\title{
LA ORGANIZACIÓN MICROESTRUCTURAL DE LOS VERBOS DEL DAELE: EL USO DE ETIQUETAS SEMÁN- TICAS COMO RECURSO DIDÁCTICO
}

\author{
Rejane Bueno ${ }^{1}$ \\ Universidad Federal de la Integración Latinoamericana \\ buenorejane@gmail.com
}

\begin{abstract}
Resumen: La organización de los materiales que componen la microestructura de los verbos debe tener en cuenta los diferentes aspectos inherentes a esta categoría, tales como la polisemia o las restricciones de combinatoria léxica y sintáctica. Si el repertorio lexicográfico va dirigido a usuarios aprendices de lengua extranjera estos matices cobran especial relevancia no sólo por su especificidad lingüística, sino también por una necesidad de organización didáctica de tales informaciones. El "Diccionario de aprendizaje del español como lengua extranjera" (DAELE) ${ }^{2}$ es un diccionario que por las características propias del tipo de usuario al que va dirigido tiene la nomenclatura restringida, motivo por el que se han introducido las etiquetas semánticas en la elaboración de los artículos lexicográficos. Este recurso nos permitirá agrupar el número de entradas y distinguir entre diferentes acepciones y subacepciones teniendo en cuenta aspectos como la información semántica y la sintáctica y combinatoria. En este trabajo analizaremos la pertinencia de la introducción de las etiquetas semánticas en los verbos de DAELE y también el tipo de materiales que caracterizan dichas etiquetas. El objetivo es observar si este recurso constituye de hecho un buen criterio para la redacción de los artículos lexicográficos verbales y si su empleo en este tipo de repertorio es un buen recurso didáctico para el aprendizaje de la lengua española.
\end{abstract}

Palabras clave: Lexicografía pedagógica, Representación lexicográfica de verbos, Etiquetas semánticas. 


\title{
THE MICROSTRUCTURAL ORGANIZATION OF DAE- LE VERBS: USAGE OF SEMANTIC LABELS AS A DIDACTIC RESOURCE
}

\begin{abstract}
The organization of the materials that compose the verbs microstructure must account for the different aspects which are inherent to this category, such as polysemy or the word and syntactic restrictions. These nuances are especially relevant for the lexical repertoire that is tailored to learning users of foreign language, both for their linguistic specificity and the necessity for didactic organization of such information. The "Dictionary of learning Spanish as a foreign language" (DAELE) 11 is a dictionary tailored to the characteristics of a specific user with restricted nomenclature, thus the introduction of semantic tags in the preparation of lexical units. The semantic tags are a lexicographic resource that allows us group the number of entries and distinguish between different senses and subsenses considering aspects such as word combinations and syntactic information. In this paper we analyze the relevance of introducing semantic tags in the DAELE verbal entries and also the type of materials characterizing such tags. The purpose was to examine whether such resource is a good criteria for writing the verbal lexical units and if its use in this kind of repertoire is a good didactic tool for the leaning of the Spanish language.
\end{abstract}

Keywords: Pedagogical lexicography, lexicography representation of verbs, semantic tags.

\section{Antecedentes}

El Diccionario de Aprendizaje del Español como Lengua Extranjera (DAELE) 4 es un diccionario de lengua española de nueva planta que se está elaborando en el seno de varios proyectos de investigación del Grupo Infolex, en la Universitat Pompeu Fabra (Barcelona/España) 5. Uno de los objetivos de este repertorio es construir un puente entre las reflexiones sobre la lingüística aplicada a la enseñanza de LE y la lexicografía pedagógica práctica.

En este trabajo se abordarán algunas de las reflexiones que han tenido lugar durante la redacción de los artículos lexicográficos de los verbos. Debido a la naturaleza altamente polisémica de estas voces, asociada a la necesidad de una nomenclatura restringida, 
característica propia de un repertorio lexicográfico dirigido a usuarios aprendices de lengua, se ha decidido utilizar en la redacción de los artículos las etiquetas semánticas con el objetivo de simplificar y optimizar la organización de las entradas. Se presentará la organización macro y micorestructural del DAELE y luego el tipo de etiquetas semánticas que se ha utilizado en el diccionario.

\section{EI DAELE}

El Diccionario de Aprendizaje del Español como Lengua Extranjera es un repertorio lexicográfico monolingüe, dirigido a estudiantes de ELE de nivel avanzado (B2-C), pensado prioritariamente para la codificación en lengua española. Es el primer diccionario de ELE que ha sido concebido para Internet y que solo se ofrece en este soporte, lo que permite un work in progress y permite ofrecer una gran cantidad de información y diferentes posibilidades de consultarla. La opción extendida y la reducida. La primera contiene todos los ejemplos disponibles para cada una de las subacepciones y también incluye notas de uso, en algunos casos, la segunda, incluye menos ejemplos, normalmente dos o tres y no muestra las notas. La información de los lemas se ha organizado con la base de datos lexicográfica Tshwanelex y el análisis de los aspectos léxicos y de la combinatoria se ha llevado a cabo en varios corpus ${ }^{6}$. Actualmente están disponibles en la web del DAELE 363 verbos para la consulta en línea.

\subsection{La macroestrctura del DAELE}

La confección del lemario de los verbos se ha definido a través de criterios de frecuencia y uso en corpus constituidos de diferentes tipos de textos, a partir de los que se ha elaborado un listado de los 1380 verbos más frecuentes de la lengua española (L1380)7. Se ha llevado a cabo una agrupación semántica a través de las relaciones 
hiponímicas e hiperonímicas para facilitar la redacción de los artículos lexicográficos a partir de grupos de significados cercanos.

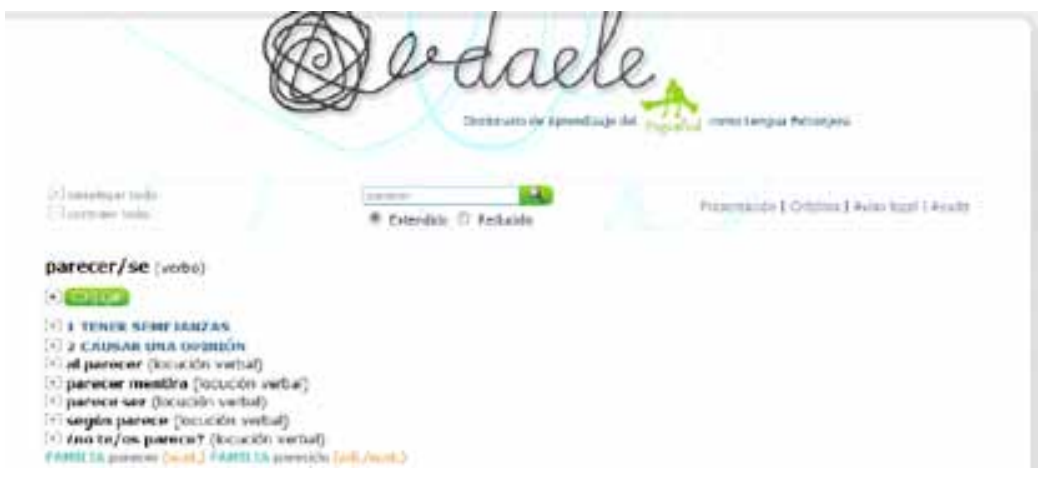

Figura 1. Visión de la página del DAELE por defecto

\subsection{La microestructura del DAELE}

La microestructura del DAELE se organiza a partir de criterios semánticos para la separación de las acepciones y supeditado a ellos queda la información sintáctica. Siempre que hay más de una acepción cada una de ellas va encabezada por una etiqueta semántica.

Cada acepción se considera como un macro significado y en su interior se agrupan las subacepciones específicas, que en algunas ocasiones se desglosan en patrones argumentales distintos según las alternancias o variantes sintácticas. Para evitar formalismos en las definiciones, en la marcación de los argumentos verbales y en la presentación de las construcciones sintácticas básicas se ha elegido la definición natural. En las figuras abajo pueden observarse capturas de pantalla en la versión reducida y extendida. 


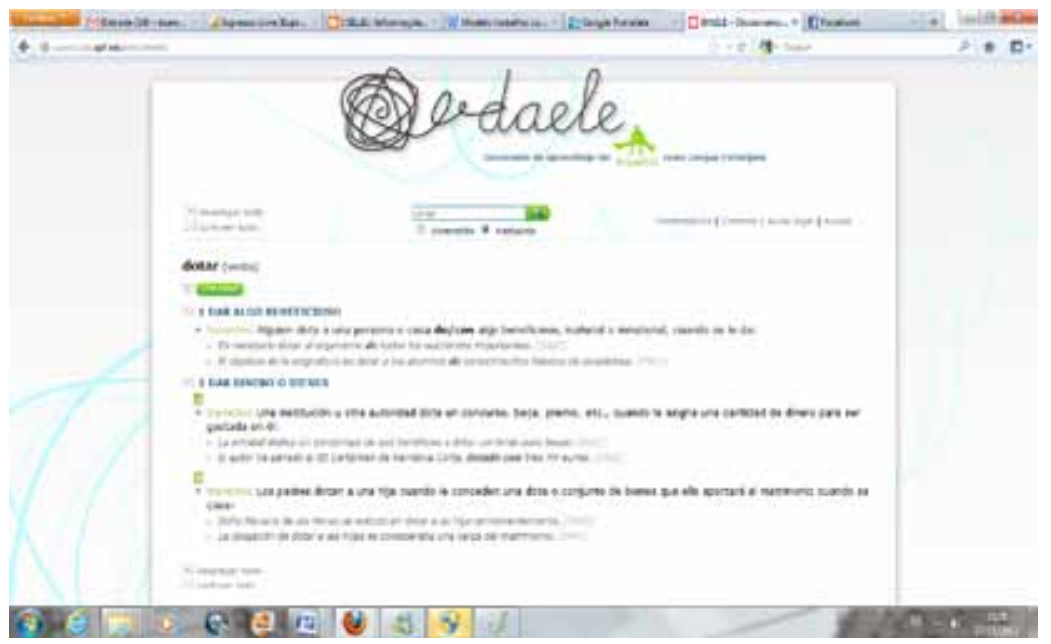

Figura 2. Visión de la página del DAELE en la versión reducida

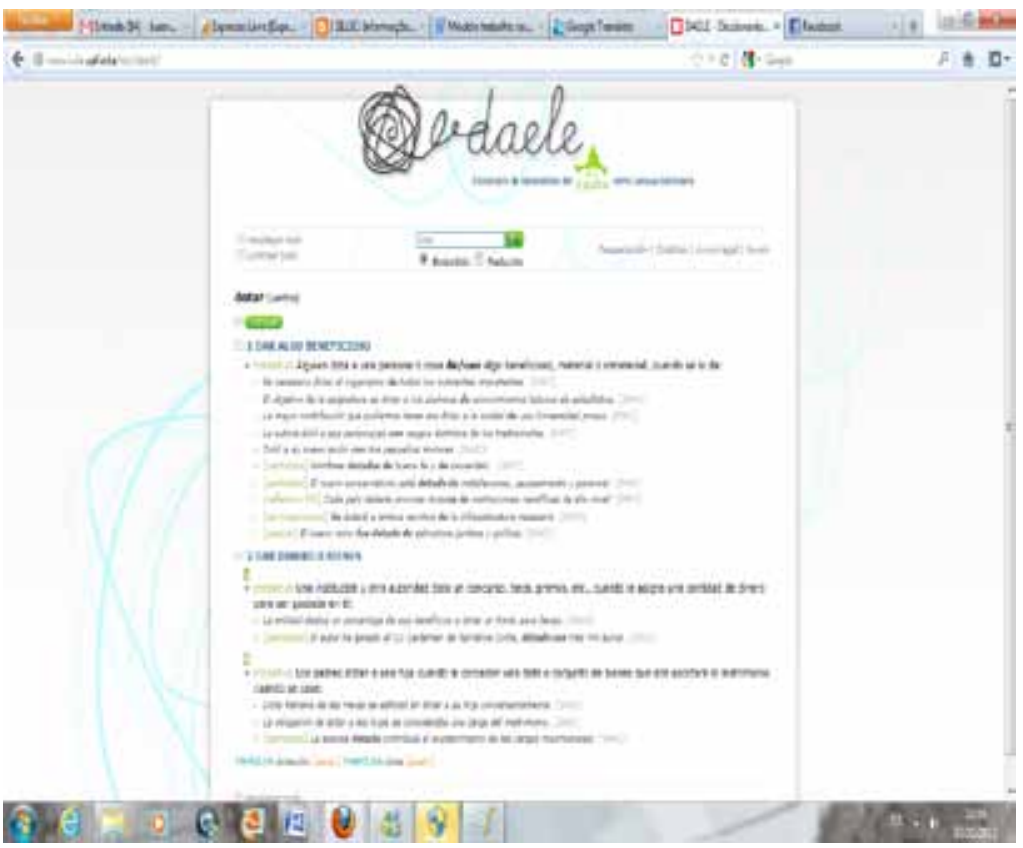

Figura 3. Visión de la página del DAELE en la versión extendida 
Se puede observar en la figura 3 la misma entrada en su versión extendida, con casi toda la información desplegada, excepto la conjugación verbal. Puede observarse que la segunda acepción está desglosada en dos subacepciones que van acompañadas de exhaustivos ejemplos recopilados de diferentes corpus.

Para la información sintáctica, además de ofrecer las marcas de categoría y subcategoría gramaticales, se ofrecen indicaciones sobre la construcción sintáctica delante de los ejemplos, se marca el subjuntivo que requieren las subordinadas del verbo y si son de sujeto o de objeto, estructuras sintácticas muy fijadas relacionadas con la subacepción etc. Se señalan con negrita algunos elementos, como las preposiciones regidas o ciertos pronombres que acompañan al verbo. Por último, el DAELE ofrece también, cuando se considera necesario, la combinatoria léxica de nombres y adverbios, la familia etimológica de palabras, los sinónimos y antónimos de un determinado significado, siempre y cuando se considere que esta información puede ayudar al usuario a comprender y/o utilizar adecuadamente el lema.

\section{Las Etiquetas semánticas y el DAELE: teoría y práctica}

Con la finalidad de atender a la necesidad de las múltiples informaciones necesarias para la descripción lexicográfica de un verbo, la necesidad real del usuario aprendiz, teniendo en cuenta su capacidad para extraer la información lingüística de una entrada lexicográfica, se ha decidido que el contenido semántico de las entradas es preeminente al sintáctico. Esta es la decisión básica sobre la cual se apoya el uso de las etiquetas semánticas. La utilización de este recurso no es novedosa en la lexicografía de aprendizaje en diccionarios de lengua inglesa, sin embargo en diccionarios de lengua española todavía no es un recurso conocido ${ }^{8}$.

Se definen las etiquetas semánticas del DAELE como descriptores orientativos que encabezan las acepciones y en torno a las que 
se organizan los lemas polisémicos y que contienen los significados nucleares de los verbos, permitiendo que el usuario no tenga que recorrer todas las definiciones hasta encontrar la que busca9. Además, visa permitir la distinción clara entre diferentes acepciones y subacepciones sin dejar de tener en cuenta los aspectos como la información sintáctica y la combinatoria. De esta forma se simplifica y se organiza jerárquicamente la redacción del artículo lexicográfico, concentrándose en las relaciones paradigmáticas que se establecen entre los verbos y sus acepciones.

Béjoint (2010, p.176) señala algunas cuestiones importantes que se deben tener cuenta en la utilización de este tipo de descriptores:

a. Se supone que el usuario ya tiene alguna idea del significado de la palabra que está buscando;

b. Son de naturaleza heterogénea (pueden ser colocaciones, sinónimos, hiperónimos, nombres de dominio, etc.;

c. Son especialmente importantes en los diccionarios electrónicos ya que la cantidad de información que un usuario puede ver está limitada por la pantalla y por lo tanto un resumen de la información es útil. ${ }^{x}$

La heterogeneidad tipológica de las etiquetas apuntada por el autor, indica la dificultad en la redacción de estos descriptores, posiblemente agudizada por las diferencias entre clases de palabras y también por las diferencias entre unidades de una misma clase. En este contexto, cabe reforzar la complexidad inherente a la categoría del verbo en cuanto a la especificación del significado (mucho menos específica que los nombres, por ejemplo) así que el descriptor que se utilice para guiar la consulta a entradas de este tipo tiene que aportar elementos que permitan distinguir un determinado significado frente a otro. Por ejemplo, el lema combinar/se en el DAELE, en la segunda acepción aparece con la etiqueta " 2 pasarse el balón”, en este caso la presencia del argumento es fundamental en 
el descriptor para que se pueda entender el significado específico del lema frente a *pasarse el vaso/el libro/cualquier objeto, que no se realizan con el lema combinarse, hay una restricción de combinación léxica que es inherente al verbo, por lo tanto debe figurar en una etiqueta que dé cuenta de su significado nuclear. Sobre la necesidad de distinción de los significados a partir de los descriptores semánticos, DeCesaris (2012, p.533) matiza: "In fact, some signposts in monolingual learner's dictionaries are reminiscent of contextual information included in large bilingual dictionaries to disambiguate senses. " .

Battaner (2011, p: 144) explica el proceso de redacción de las etiquetas semánticas en el DAELE y hace hincapié en la 'escurridiza' intensión de los verbos sin sus argumentos correspondientes, da como ejemplo el verbo correr, en el que únicamente se fijan las extensiones cuando se delimitan los argumentos, por ejemplo, el hombre/el leopardo/el tiempo corre, pero *un libro/el cielo corre $^{12}$. Se ha defendido que la etiqueta semántica sea otro verbo, sin embargo la redacción de descriptores semánticos para palabras altamente polisémicas implica tener en cuenta las restricciones léxicas, sintácticas y combinatoria de los argumentos, así que se ha decidido incluirlos siempre y cuando sean fundamentales para la comprensión de los significados nucleares de las unidades descritas en el repertorio.

Por todo lo que se ha dicho hasta ahora, abogamos por el uso de las etiquetas semánticas. No hay dudas que la utilización de estos descriptores en diccionarios para el aprendizaje de lenguas extranjeras ayuda a guiar al usuario inexperto en consultas a este tipo de materiales, ya que facilita la búsqueda y organiza las informaciones semánticas sin dejar de tener en cuenta la sintaxis de los lemas polisémicos (como lo son los verbos por defecto). De esta forma se tendrá una menor cantidad de acepciones encabezada por la información semántica que contiene los significados nucleares del verbo, mientras que la sintáctica y combinatoria queda supeditada a la semántica y aparecerán en forma de subacepciones. 
Nuestra intención en este trabajo es mostrar cómo se ha llevado a cabo la inclusión de etiquetas semánticas partiendo del DAELE como un primer intento de sistematizarlo en la práctica lexicográfica en lengua española, con lo cual observaremos cuáles son los tipos de etiquetas que se ha utilizado hasta el momento teniendo en cuenta dos postulados de la técnica lexicográfica:

a. lo que Landau (2001) ha desarrollado como la necesidad de coherencia interna en los patrones de descripción lexicográfico, $y$

b. lo que Jackson (2002) he definido como la necesidad de reconocer en el diccionario un programa constante de informaciones.

Bajo esa perspectiva, el conjunto de informaciones lingüísticas en un artículo lexicográfico cobra importancia no únicamente por su valor lingüístico en sí, sino por la forma estratégica como está dispuesto, además esta información debe ser coherente y constante durante toda la descripción, ya sea en presencia, o ausencia. Estos serán los principales puntos que nos interesan observar en la tipología que comentamos a continuación.

\subsection{Las etiquetas semánticas del DAELE}

Se puntualizarán los criterios que permiten distinguir y caracterizar los diferentes tipos de etiquetas semánticas utilizadas en el DAELE, el objetivo es diseñar una tipología que permita entender el programa constante de informaciones del DAELE, así como observar la coherencia interna de los descriptores semánticos. Se han observado las 363 entradas verbales ya redactadas y colgadas en la web del DAELE teniendo en cuenta cada una de las acepciones y subacepciones. Se ha llevado a cabo un análisis general del listado de los lemas con las etiquetas y sus definiciones completas, 
de modo que nos permitiera averiguar qué tipo de glosas se utiliza para explicar semánticamente cada una de las voces definidas y qué tipo de descriptor semántico encabeza cada una de las glosas. Se ha llegado a reconocer hasta seis tipos distintos de etiquetas:

1. sinonímicas,

2. hiperonímicas,

3. parafrásticas

4. parafrásticas con argumento

5. exploitaition

6. instructivas

Tal como se explican a continuación.

\begin{tabular}{|c|c|c|}
\hline Tipo de etiqueta & Definición & Ejemplos \\
\hline \multirow[b]{2}{*}{ Sinonímica } & \multirow[b]{2}{*}{$\begin{array}{l}\text { La etiqueta es un sinóni- } \\
\text { mo del lema definido. }\end{array}$} & Avasallar [agobiar] \\
\hline & & $\begin{array}{l}\text { Estimar } \\
\text { [apreciar, valorar] }\end{array}$ \\
\hline
\end{tabular}

Es importante señalar que no se trabaja con una noción de sinonimia absoluta, sino que se apuesta por una aproximación de significados entre las unidades léxicas, lo que Seco (1977, p.32) ha nombrado "igualdad a efectos prácticos". Para observar la sinonimia, además de la consulta al DAELE, se ha consultado dos diccionarios: el DRAE y el DUEAE, en los que se han ido contrastando las definiciones de ambos repertorios a la vez y siempre que había aproximación mayoritaria entre los significados, se consideron sinónimos. 


\begin{tabular}{|l|l|l|}
\hline Tipo de etiqueta & Definición & Ejemplos \\
\hline \multirow{2}{*}{ Hiperonímica } & $\begin{array}{l}\text { La etiqueta es una unidad léxica } \\
\text { que se considera un hiperónimo } \\
\text { del lema definido. }\end{array}$ & $\begin{array}{l}\text { Abrigar } \\
\text { [proteger] }\end{array}$ \\
\cline { 3 - 3 } & & $\begin{array}{l}\text { Luchar } \\
\text { [enfrentarse] }\end{array}$ \\
\hline
\end{tabular}

Para caracterizar las etiquetas semánticas hiperonímicas se observaron dos aspectos: la presencia del descriptor en la definición del propio lema, o la presencia de la unidad en el listado de hiperónimos que se ha utilizado en la confección de la nomenclatura del DAELE. Tal como se explicó anteriormente, el lemario del DAELE surge de una agrupación semántica que se basa en las relaciones hiperónimo-hipónimo en la que se contrastaron a través de medios informáticos lemarios y definiciones de dos diccionarios generales de lengua española.

\begin{tabular}{|l|l|l|}
\hline Tipo de etiqueta & Definición & Ejemplos \\
\hline & & $\begin{array}{l}\text { Abrir/se } \\
\text { [mostrarse re- } \\
\text { ceptivo] }\end{array}$ \\
\cline { 2 - 2 } Parafrástica & $\begin{array}{l}\text { La etiqueta es una paráfrasis ver- } \\
\text { bal del lema definido. } \\
\text { [moverse rít- } \\
\end{array}$ & $\begin{array}{l}\text { micamente] } \\
\text { maldar }\end{array}$ \\
\hline
\end{tabular}

Se ha tenido en cuenta si la etiqueta se desdobla en un sintagma en el que se observa una paráfrasis verbal del lema que se define. Lo importante es que en este tipo de etiquetas no se observe ninguna información semántica explícita sobre los argumentos verbales. 


\begin{tabular}{|c|c|c|}
\hline Tipo de etiqueta & Definición & Ejemplos \\
\hline \multirow[t]{2}{*}{$\begin{array}{l}\text { Parafrástica con } \\
\text { argumento }\end{array}$} & \multirow{2}{*}{$\begin{array}{l}\text { La etiqueta es una paráfrasis } \\
\text { verbal del lema y además in- } \\
\text { cluye información semántica } \\
\text { sobre alguno de los argumentos } \\
\text { obligatorios del verbo. }\end{array}$} & $\begin{array}{l}\text { Oprimir } \\
\text { [ejercer } \\
\text { presión física] } \\
\text { [ejercer } \\
\text { presión moral] }\end{array}$ \\
\hline & & $\begin{array}{l}\text { Segar } \\
\text { [cortar cereal] }\end{array}$ \\
\hline
\end{tabular}

La etiqueta parafrástica con información del argumento, es la que se desdobla en un sintagma que es una paráfrasis verbal del lema y que además aporta alguna información semántica sobre alguno de los argumentos verbales, ya sean internos, o externos. Como se ha discutido, este recurso se utilizará siempre y cuando dicha información sea crucial para que el usuario emplee el lema de forma inequívoca, como observamos en el segundo ejemplo, segar, debe incluir la información sobre qué es pasible de segarse/ cortarse, ya que sin esa información un usuario extranjero podría perfectamente construir la sentencia *Necesito segarme el pelo.

\begin{tabular}{|c|c|c|}
\hline Tipo de etiqueta & Definición & Ejemplos \\
\hline \multirow[t]{2}{*}{ Exploitation } & \multirow{2}{*}{$\begin{array}{l}\text { La etiqueta indica una acepción } \\
\text { que es un uso figurado o estilís- } \\
\text { tico del lema. }\end{array}$} & $\begin{array}{l}\text { Cortar/se } \\
\text { [mostrarse } \\
\text { tímido] }\end{array}$ \\
\hline & & $\begin{array}{l}\text { Comer } \\
\text { [irritar o mo- } \\
\text { lestar] }\end{array}$ \\
\hline
\end{tabular}


El concepto de exploitation se remonta a Hanks (en prensa), que opone este concepto al de norms, siendo esta el uso ad hoc, normal de una palabra, frente al de exploitation como el uso novedoso, estilístico del lenguaje, la creación de 'nuevos significados ad hoc' en el lenguaje, como se observa en la cita:

Lexical exploitation is a dynamic mechanism of language, used to create new meanings ad hoc and to say old things in new ways. [...] An exploitation is a deliberate departure from an established pattern of normal word use, either in order to talk about new or unusual things or in order to say old things in a new, interesting, or unusual way. [...] Exploitations are distinguished from alternations ${ }^{13}$. (Hanks, en prensa, p: 161)

Aunque el objetivo de un diccionario para hablantes no nativos no es recopilar usos estilísticos o no normativos de una lengua, algunas de las acepciones de los lemas que se etiquetaron con marcadores de este tipo son bastante comunes en el lenguaje al que los usuarios tendrán acceso, por eso se ha decidido registrar en el DAELE. Además, como todos los ejemplos se han extraído de corpus, se matiza la pertinencia de estas acepciones en el diccionario.

\begin{tabular}{|l|l|l|}
\hline Tipo de etiqueta & Definición & Ejemplos \\
\hline \multirow{3}{*}{ Instructiva } & $\begin{array}{l}\text { La etiqueta presenta una } \\
\text { orientación directa cuanto al } \\
\text { uso del verbo. }\end{array}$ & $\begin{array}{l}\text { Hacer/se } \\
\text { [verbo que sus- } \\
\text { tituye } \\
\text { a otros] }\end{array}$ \\
\cline { 2 - 3 } & & $\begin{array}{l}\text { Tener } \\
\text { [verbo de apoyo] }\end{array}$ \\
\hline
\end{tabular}


Estos descriptores se han llamado también "metaetiquetas", ya que presentan más que información semántica sobre el lema, pero sí una instrucción de cómo debe usarse esta unidad léxica. Es un recurso que se ha utilizado como última alternativa a una descripción semántica propiamente dicha. Se ha empleado minoritariamente en el DAELE y siempre con verbos auxiliares/soporte.

\subsection{Apreciación de la tipología de etiquetas en el DAELE}

Se ha analizado la distribución tipológica de las etiquetas de los 363 verbos definidos en el DAELE hasta el momento, y ha resultado cuantitativamente la siguiente tabla:

\begin{tabular}{|l|l|}
\hline Etiqueta & Porcentaje \\
\hline Parafrásticas & $40,47 \%$ \\
\hline Hiperonímicas & $21,99 \%$ \\
\hline Parafrásticas con argumento & 20,95 \\
\hline Sinonímicas & $14,25 \%$ \\
\hline Exploitation & $2,13 \%$ \\
\hline Instructivas & $0,71 \%$ \\
\hline
\end{tabular}

Hasta el momento la mayoría de las etiquetas del DAELE son parafrásticas, seguidas de las hiperonímicas, luegoestán las parafrásticas con informaciones sobre los argumentos $\mathrm{y}$, en menor cantidad, las sinonímicas. Con baja representatividad están las exploitations y las instructivas. Estos datos reflejan la relación que hay entre las etiquetas semánticas y la definición de verbos, ya que una paráfrasis es mucho más explicativa que un sinónimo, que puede generar circularidad en la definición. Un sinónimo, a su vez, es menos aclarador que un hiperónimo que por contener todos los 
semas de su hipónimo puede ser más aclarador al usuario porque es más amplio en su significación, pero, por otro lado, es menos preciso. Las etiquetas parafrásticas con argumento aparecen en tercer lugar debido a que estos descriptores semánticos deben ser lo más breves posible, ya que son solamente una directriz que guiará el usuario hacia el significado que se explicita en la glosa definidora. De ahí, cabe matizar que el papel de la etiqueta semántica no es precisar la significación del verbo, sino guiar el usuario hacia el significado más cercano frente a las demás opciones.

Si retomamos el ejemplo de segar, podemos averiguar la coherencia de interna en la redacción de las etiquetas. En este caso es fundamental que se aclare ya en la etiqueta la especificidad del argumento interno del verbo, ya que en este caso dicha selección argumental limita las posibilidades de combinación léxica del verbo en esta acepción. Aún verificando los aspectos de la coherencia interna de las etiquetas, observemos un lema que lleva una etiqueta de tipo explotation:

\section{Cortar}

5 [ser premiado] Un torero corta una oreja o el rabo cuando consigue esta parte del toro que ha lidiado, como premio por la buena faena realizada.

Se puede observar que esta es la acepción número cinco del lema, las cuatro anteriores anticipan significados más comunes de la lengua. Esta acepción además está diatópicamente marcada, ya que se refiere al léxico del toreo, más característica de la variedad peninsular, lo que se podría decir que es un significado 'nuevo' de la lengua a partir de las mismas estructuras. Este es el tipo de fenómeno lingüístico que Hanks (en prensa) ha nombrado exploitation.

A modo de conclusión, se puede comprobar que hay un programa constante de informaciones en cuanto a la facilitación de acceso a la información semántica en el interior de la microestructura de los artículos lexicográficos del DAELE, ya que en todos los lemas 
con más de una acepción hay una etiqueta semántica. Estos descriptores semánticos son coherentes no sólo internamente, siempre que se han puesto en contraste con las glosas definidoras de las voces, mostrándose aclaradores y simplificadores de los significados más específicos, sino que son coherentes con lo que es la técnica de definición que se aconseja para los verbos en diccionarios de aprendizaje de lengua extranjera. Se pudo evitar circularidades a través de sinonimias, generalidades a través de hiperonimias y explicitar los diferentes usos a través de paráfrasis sencillas, cortas y con la información argumental siempre y cuando es necesaria, además de describir algunos usos estilísticos fundamentales para convertir la producción lingüística del extranjero en una producción natural.

\section{Conclusiones}

Durante la ejecución de este trabajo se ha podido percibir que hay en la redacción de los artículos del DAELE una preocupación sistemática en hacer las descripciones lexicográficas de los aspectos lingüísticos bajo las directrices de la lexicografía y, además, de hacerlas de una forma más asequible al usuario aprendiz, respondiendo a uno de los principales postulados de la lexicografía pedagógica. La organización microestructural de los artículos verbales en torno a las etiquetas semánticas es un buen ejemplo de ello, es un importante recurso para facilitar la consulta al diccionario, o como describió Lew (2010) access facilitating devices. Se ha observado que las etiquetas semánticas simplifican y acortan la redacción de los artículos de lemas polisémicos, como lo son los verbos inherentemente, organizan las acepciones a partir de semántica y además permiten incluir la información sintáctica y combinatoria en un segundo plan de importancia. La organización de los lemas por estos significados nucleares hacen más evidentes las distinciones semánticas entre las acepciones convirtiendo el uso de las etiquetas semánticas es uno de los principales recursos didácticos 
que puede auxiliar al usuario en la consulta de artículos más largos y complejos, como muchos de los verbos.

Al analizar el tipo de etiquetas semánticas se han observado seis tipos diferentes, siendo la más usual la etiqueta parafrástica, que consiste en una breve explicación del lema, evitando circularidades y obviedades que perjudican la consulta del usuario extranjero. Llaman la atención las etiquetas parafrásticas con información semántica sobre los argumentos verbales, que pueden ser de gran ayuda para la distinción entre lemas que tienen algún tipo de restricción de combinación léxica o incluso sintáctica. Cabe poner de relieve las etiquetas que registran algunos usos estilísticos, las que aquí llamamos exploitation, estos usos deben aparecen en un diccionario para aprendices extranjeros siempre y cuando sean de real utilidad para este usuario, tal y como se hizo en este repertorio.

En el programa constante de informaciones del DAELE las etiquetas semánticas están presentes en todos los artículos con más de una acepción, además se comprobó la constancia de separación de las acepciones por criterios semánticos, dejando supeditadas a las subacepciones las alternancias sintácticas. Asimismo las etiquetas se han demostrado coherentes internamente porque acompañan las recomendaciones de la lexicografía teórica en lo que respecta la definición de verbos y poseen patrones de redacción uniformes que permiten agruparlas de forma más o menos uniforme. Por último cabe decir que este recurso tiene un fuerte matiz didáctico porque permite evitar formalismos a la hora de definir unidades léxicas complexas como los verbos. Este hecho representa un gran avance en la lexicografía en lengua española y contribuye para que este tipo de repertorio lexicográfico funcione como una verdadera herramienta de aprendizaje del español, haciendo la conexión entre la lexicografía teórica y la lingüística aplicada a la enseñanza de lenguas. 


\section{Notas}

1. Mestre en Letras UFRGS (Lexicografía y Terminología), profesora en la Universidad Federal de la Integración Latinoamericana (UNILA) e investigadora del grupo INFOLEX - Universitat Pompeu Fabra/España.

2. Este diccionario se está llevando a cabo en el interior de los proyectos de investigación del Grupo Infolex, en la Universidad Pompeu Fabra - España.

3. This dictionary has been developed by the research projects of Infolex, from the Pompeu Fabra University (Spain).

4. http://www.iula.upf.edu/rec/daele.

5. «Las categorías nombre y adjetivo en el DAELE (Diccionario de aprendizaje del español como lengua extranjera)»(HUM2006-07898/FILO), «Las categorías del verbo y del adverbio en el DAELE (Diccionario de Aprendizaje del español como lengua extranjera)» (HUM2006-06982/FILO), «Agrupación semántica y relaciones lexicológicas en el diccionario» (HUM2009-07588/FILO). El DAELE recibe también financiación de la Fundación Comillas.

6. El Spanish Web Corpus por medio de la herramienta Sketch Engine, el Corpus del español y los corpus disponibles entre las herramientas del IULA (Institut Universitari de Lingüística Aplicada).

7. La explicación detallada de como se ha constituido este listado de frecuencia está en Battaner y Renau (2010).

8. Battaner (2011, p: 143) menciona al menos tres diccionarios de lengua inglesa que utilizan las etiquetas semánticas en sus descripciones: el CALD, LDCE y MEDO.

9. En la lexicografía de aprendizaje de lengua inglesa se ha nombrado a ese tipo de recurso como "access facilitating devices" (LEW, 2010). 
10. Este último punto es especialmente relevante para DAELE, visto que es un diccionario en línea.

11. De hecho, algunas etiquetas en diccionarios monolingües de aprendizaje son una reminiscencia a la información contextual que está incluída en grandes diccionarios bilingües de forma a eliminar la ambigüedad de los sentidos. (traducción nuestra)

12. Para la definición de los argumentos de los verbos del DAELE se ha llevado a cabo una minuciosa descripción lingüística de los patrones sintáctico, semántico y combinatorio de las unidades a partir del análisis de corpus.

13. Explotación lexica es un mecanismo dinamico del lenguaje (de la lengua), que se usa para crear nuevos significados ad hoc y para decir las cosas antiguas de nueva manera. [...] Una explotación es una desviación deliberada de un patrón establecido de uso normal de las palabras, sea para hablar de cosas nuevas o inusuales, o para decir cosas antiguas de una nueva, interesante o una forma inusual. [...] Explotaciones se distinguen de las alternancias.

\section{Referencias}

BATTANER, P.; S. TORNER. La polisemia verbal que muestra la lexicografía. En: AZORÍN, D. et al. (ed.). Actas del II congreso internacional de lexicografía hispánica. El diccionario como puente entre lenguas y culturas del mundo. Alicante: Taller Digital y Fundación Biblioteca Virtual Miguel de Cervantes, 2008, p. 204-217.

BATTANER, P.; RENAU, I. Agrupación semántica de verbos de un DAELE a través de sus relaciones hiperonímicas. La lexicografía en su dimensión teórica. Málaga: Servicio de Publicaciones de la Universidad de Málaga, 2010, p. 543560 . 
BÉJOINT, H. The Lexicography of English. Oxford: OUP, 2010.

BUENO, Rejane. El diccionario de aprendizaje del español como lengua extranjera (DAELE): una herramienta para la enseñanza del verbo. En: Horizontes de Brasil: escenarios, intercambios y diversidad. Barcelona: Ediciones APEC (Asociação de Pesquisadores Brasileiros da Catalunha). 2011, p. 1948-1958.

. El Diccionario de aprendizaje de español como lengua extranjera (DAELE): una nueva herramienta en el aprendizaje de LE. Una mirada hacia la representación de la categoría del verbo. En: Domínios de Lingu@gem 5(2). Uberlândia: Universidade Federal de Uberlândia, 2011, p. 202-216.

COWIE, A. P. English Dictionaries for Foreign Learners: A History. Oxford: OUP, 1999.

DECESARIS, J. On the nature of signposts. En: PROCEEDINGS OF THE 15TH EURALEX INTERNATIONAL CONGRESS. Oslo: University of Oslo, 2012, p. 532-540.

GARRIGA ESCRIBANO, Cecilio. La microestructura del diccionario: las informaciones lexicográficas. En: MEDINA GUERRA, Antonia M. (org.). Lexicografía Española. Barcelona: Ariel Lingüística, 2003, p. 103-125.

HANKS, P. Lexical Analysis: norms and exploitaition. Expuesto en un curso organizado por el grupo Infolex - IULA, mayo/2009, (en prensa).

HARTMANN, R.R.K. Teaching and researching lexicography. London: Longman, 2001.

JACKSON, Howard. Lexicography. London: Routledge, 2002.

LANDAU, Sidney. Dictionaries: The art and craft of lexicography. Cambridge: CUP, 2001. 
LEW, R. Users Take Shortcuts: Navigating Dictionary Entries. En: Dykstra, A.; T. Schoonheim (eds.). Proceedings of the XIV Euralex International Congress. Ljouwert: Afûk, 2010, 1121-1132.

MEDINA GUERRA, Antonia M. Medina (org.). Lexicografía Española. Barcelona: Ariel Lingüística, 2003.

. La Microestructura del diccionario: la definición. En: GUERRA, Antonia M. Medina (org.). Lexicografía Española. Barcelona: Ariel Lingüística, 2003, p. 127-146.

RUNDELL, M. Recent Trends in English Pedagogical Lexicography. En: International Journal of Lexicography, $\mathrm{n}^{0} 11 / 4,1998,315-342$.

SECO, M. Problemas formales de la definición lexicográfica. Estudios de Lexicografía española. $2^{\mathrm{a}}$ ed. Madrid, Gredos, 1977, p. 25-46.

. El ‘contorno' en la definición lexicográfica”. En: Estudios de Lexicografía española. $2^{\text {a }}$ ed. Madrid, Gredos, 1977, p. 47-58.

\section{Diccionarios}

[CALD] Cambridge Advanced Learner's Dictionary. (en línea) http://dictionary. cambridge.org/

[DAELE] Battaner, P.; J. DeCesaris (dirs.). Diccionario de Aprendizaje de Español como Lengua Extranjera, en proceso de redacción, Institut Universitari de Lingüística Aplicada, Universitat Pompeu Fabra. < http://www.iula.upf.edu/ $\mathrm{rec} /$ daele $>$

[DRAE] Real Academia Española (en línea). Diccionario de la lengua Española, Actualización para la $23^{\mathrm{a}}$ ed., < http://www.rae.es/rae.html>

[DUE] Moliner, M. (1967) Diccionario de uso del español. Madrid, Gredos, $2^{\text {a }}$ ed, 2 vols. Ed. electrónica en CD Rom. 
[DUEAE] Diccionario de Uso del Español de América y España, VOX, Spes Editorial 2002, CD Rom 2003.

[LDCE] Longman Dictionary of Contemporary English, (en línea) < http://www. ldoceonline.com/search/ >

[MEDO] Macmillan English Dictionary Online < http://www.macmillandictionary.com>

Recebido em 16/12/2012 Aceito em 29/06/2013 